\title{
Investigating work and learning through complex adaptive organisations
}

\author{
Abstract \\ Purpose \\ This article outlines an empirical study of professionals' experiences of work and learning in \\ organisations. The study adopts and expands complexity theory in the workplace learning \\ literature, specifically, complex adaptive systems which forms the basis of an innovative \\ conceptual framework for explaining professionals' experiences of work and learning.

\section{Design/methodology/approach} \\ Semi-structured interviews were conducted with fourteen professionals from a variety of \\ organisations, industry sectors, and jobs within Australia. The transcripts were subjected to \\ an adapted phenomenographic analysis and an analysis using the complex adaptive \\ organisations conceptual framework.
}

\section{Findings}

The findings show that professionals experienced learning mainly through work, where work was experienced as fluid and influenced by varying degrees of emergence, agency, complex social networks, and adaptation. Further, the greater the degree of work fluidity, the greater the impetus towards learning through work, empirically indicating that the experience of learning in contemporary organisations is entwined with work.

\section{Originality/value}

This study innovatively used the concept of complex adaptive organisations as a conceptual framework, coupled with an adapted phenomenographic methodology, to investigate individual professionals' experiences of work and learning. The adoption of complex adaptive organisations provided more rigorous way in which to adopt complexity theory. In particular, the concept of emergence provides insights into how organisational complexity influences work and, subsequently, learning and adaptation. 


\section{Introduction}

This article outlines an empirical study which investigated the question: How do individual professionals experience work and learning in complex adaptive organisations? A conceptual framework based on complex adaptive systems theory (Holland, 1995) was developed for this study, introducing the concept of complex adaptive organisations. This term is used to describe the framework as well as to differentiate organisations as human social systems from other examples of complex adaptive systems found in nature (e.g. flocks of birds, computer networks). Adopting a complex adaptive systems approach as the basis for the conceptual framework takes up the shift towards what Fenwick et al. (2011) refer to as socio-material approaches, including complexity theory. This article suggests that a more rigorous use of complexity theory, namely the more specific concept of complex adaptive systems, offers new insights into work and learning.

The article first overviews the literature on workplace learning and complex adaptive systems before outlining the gap in the literature addressed by this study. Next, the study's conceptual framework, the complex adaptive organisations conceptual framework, is outlined followed by the methodology, which uses an adapted phenomenographic approach. The article concludes by highlighting the key contributions of the study.

\section{Complexity theory and complex adaptive systems}

Complexity theory has been suggested as offering new understandings of learning and practice in the context of work (see Davis, 2012, Fenwick, 2012, Fenwick and Dahlgren, 2015, Johnsson and Boud, 2010, Lancaster, 2012, Reich and Hager, 2014). This broad term is given to a number of different areas of scientific and social science research (Mason, 2008) referring to investigations of specific types of systems called complex systems. Complex adaptive systems are specific types of complex systems containing agents which adapt their 
strategies for working within the system in order to increase their chances of success, usually through learning processes (Holland, 1995). The dynamic nature of such systems emphasises learning and adaptation as critical aspects of the system.

Originating in the physical sciences, complexity theory has been adopted by researchers in workplace learning (Fenwick et al., 2011, Fenwick, 2012), organisational theory (Tsoukas and Hatch, 2001), organisational learning (Chiva et al., 2014, Chiva et al., 2010), management (Richardson, 2008), and education (Davis and Sumara, 2006, Jacobson et al., 2016). Complexity theory offers a way in which to consider work and learning simultaneously from the perspective of collective behaviour (that is, the organisation in this study) as well as from the perspective of individuals who can learn and adapt (Jacobson et al., 2016).

The use of complexity theory is increasing within the field of workplace learning (Fenwick, 2008) offering fresh insights into the interactions between work and learning for individuals within broader organisational and societal contexts. As Hopwood (2016) argues, sociomaterial perspectives, under which category complexity has been included (Fenwick et al., 2011), "offer a basis for disrupting many features of conventional approaches to researching professional work and learning" (p. 60). The study reported in this article continues this work, which is critical in the current environment of "fluid, ever-shifting contexts" (Hopwood, 2016, p.75). Fenwick (2012) has suggested, that in order to access the insights offered by complexity, it is necessary to use the concepts rigorously rather than metaphorically so as to fully exploit their potential for explanation. This study takes up this challenge by using complex adaptive systems to frame the study, enabling a more rigorous investigation of professionals' experiences of work and learning in complex adaptive organisations. This adds to studies of work and learning by empirically indicating the entwined nature of work and learning in diverse workplaces and the important influence of 
the key elements of complex adaptive organisations, particularly emergence, adaptation, complex social networks, and agency. Adopting this more specific concept also emphasises the criticality of considering learning and adaptation when investigating professionals' experiences of work and learning.

\section{Conceptual framework}

The complex adaptive organisations conceptual framework was developed specifically for use in the study, as at the time of the analysis there were not suitable frameworks available. Although there are similar tools such as the recent Complex Systems Conceptual Framework for Learning (Jacobson et al., 2016), within the field of school education, and the adaptive/generative learning typology of Chiva et al. (2010) within organisational learning, the complex adaptive organisations conceptual framework has been specifically developed to frame studies of work and learning. This framework foregrounds emergence within organisations, taking up elements from both the workplace learning and complex adaptive systems discourses. The framework provides a way in which to consider complex organisational contexts and an opportunity to investigate of the nature of work, as well as learning, within such organisations.

The complex adaptive organisations conceptual framework is based around four key defining elements of complex adaptive organisations adapted from the literature as being most suitable for a study of professional learning in the workplace (Mitchell, 2009, Holland, 1995). The four elements are: emergence, adaptation, complex social networks, and agency.

\section{Emergence}

Emergence is a key concept within complex adaptive systems (Mitchell, 2009, Holland, $1995)$ and it has been widely adopted within workplace learning research (e.g. Reich and Hager, 2014, Hopwood, 2014, Manidis and Scheeres, 2013, Johnsson and Boud, 2010, 
Fenwick, 2012, Hopwood, 2016). The study reported here positions emergence as a defining element of organisations rather than solely as a metaphor for learning. Emergence describes how interactions within organisations contribute to patterns at the macro-level that may have very different characteristics to those of the individual elements (e.g. people, technology, spaces, etc.) and which are not able to be fully predicted from their actions and interactions (Jacobson and Wilensky, 2006, Sawyer, 2005, Reich and Hager, 2014). Emergence is not completely random (Lancaster, 2012), creating an environment of near constant change and a lack of predictability. Within complex adaptive organisations, emergence is defined as the process by which professionals interact with each other and with their context in ways which contribute to patterns within the organisation which are not able to be completely predicted or directed.

\section{Adaptation}

For complex adaptive organisations learning is the process by which the professionals adapt to the demands of their context, and to emergence, within the organisation and with reference to their relationships to the other professionals (Mitchell, 2009, Holland, 2006). Learning and adaptation are critical parts of complex adaptive organisations, which has important implications for the ways in which individuals learn and work within them. It is the acquisition of skills, knowledge and relationships (networks) by the professionals within complex adaptive organisations that make adaptation possible.

\section{Complex social networks}

Complex social networks are specific types of networks that are not completely regular and not completely random (Newman, 2010). They are often a form of small-world network (Milgram, 1967) that has a greater number of connections between colleagues within work teams or functional areas, with a lower but significant number of connections projecting 
across these areas (Watts and Strogatz, 1998). In complex adaptive organisations, professionals operate within and across interlocking networks that are highly interconnected rather than part of a rigid hierarchy.

\section{Agency}

Human agency is an important part of what sets complex adaptive organisations apart from other examples of complex adaptive systems such as flocks of birds or computers in a network and is a key defining feature of complex adaptive systems involving humans (Giddens, 1984). Agency is also an increasing focus of the workplace learning literature (see Billett, 2011, Kersh, 2015, Billett and Smith, 2010). Within complex adaptive organisations, agency is experienced as a continuum, with all professionals having a degree of agency but no one individual or group having unconstrained agency (Dietz and Burns, 1992). This is important within complex adaptive organisations which are bounded by rules and structure.

These four key defining elements of complex adaptive organisations were used as part of the data analysis, particularly when examining the nature of work in complex adaptive organisations.

\section{Methodology}

This study adopted a phenomenographic approach, which seeks to identify the different ways in which people experience aspects of their world, such as teaching or learning (Ashworth and Lucas, 2000, Marton, 1994). Phenomenography’s positioning as an empirical research method (Marton and Booth, 1997) which describes individual experiences of phenomena provided a robust but still flexible methodological basis for the study. Traditionally, phenomenography focuses on a single phenomenon, however this study investigated experiences of both work and learning. This study therefore adopted a 'hybrid' approach, 
incorporating the key elements of phenomenography (Bailey, 2015) but with one analysis for both phenomena.

Semi-structured interviews were conducted with 14 professionals; a recommended sample size for this type of study (Trigwell, 2000). A convenience sample of participants was selected using a purposive sampling approach involving a series of strategic choices about the participants and how their selection would further the aims of the research (Palys, 2008), an approach common in phenomenography (Yates et al., 2012). The participants represented nine organisations within Australia and a range of occupational levels (e.g. administration, technical, management, etc.). Participants were drawn from organisations within the professional services, member services (peak professional body), banking, retail, radio and television, scientific research, aviation, and pharmaceutical sectors. Overall there was an even representation across occupational levels and gender, with an age range of $27-54$. The interviews used a combination of direct and critical incident (Flanagan, 1954) questions and were digitally recorded before being transcribed.

\section{Findings}

Using the adapted phenomenographic approach described above, framed by the complex adaptive organisations conceptual framework, the transcripts were analysed through an iterative process over the course of one year to develop the categories of description. The complex adaptive organisations conceptual framework was used to "bracket" (Ashworth, 1999) the analysis, enabling theoretical assumptions to be defined from the outset and then put to one side to be used as part of the final stage of analysis. Through the analysis it emerged that professionals in complex adaptive organisations experienced work and learning in four interrelated ways, namely: 
(1) Learning is experienced by professionals as being through work but seldom named as such;

(2) Work is experienced by professionals as fluid and influenced by varying degrees of emergence, agency, complex social networks, and adaptation;

(3) Professionals' experiences of learning are influenced by the degree of fluidity experienced in their work;

(4) Professionals experience an organisational emphasis on structured learning.

The outcome space (Marton and Booth, 1997) illustrating these findings is shown in Figure 1.

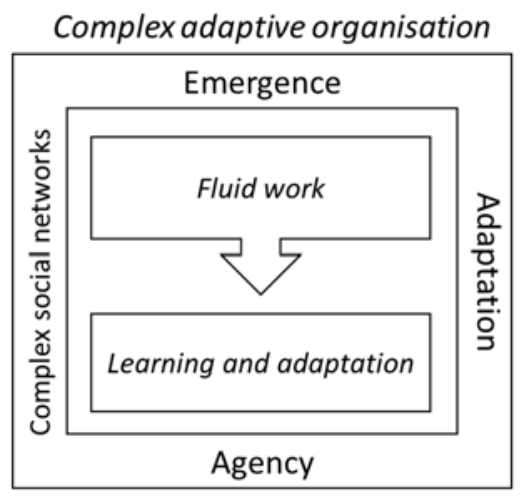

Figure 1 - The outcome space

The general themes across all categories related to the entwined relationship of work and learning experiences. As shown in Figure 1, the four elements of complex adaptive organisations influenced experiences of both work and learning, in particular work. Fluid work had a strong influence on learning in a context where learning through work was experienced as the primary mode of learning. The term 'fluid' is used to describe the experience of work that emerged from the data and, in the sense used here, is juxtaposed with the idea of work being structured and clearly planned out in advance. These connections are highlighted within the discussion of each category which follows. 
The first category which emerged from the data analysis describes how learning was primarily experienced through work but seldom named as such. A finance analyst from a government scientific organisation summarised it as,

...basically it's on the job learning and just learning as the circumstances come up.

Similarly, a manager from a member services organisation referred to, ...the things I'm learning are very much on the job, on the instant, on the fly.

These findings are aligned with existing research, which has found that much of the learning at work occurs through the practice of work (Billett, 2004, Eraut, 2007, Boud and Hager, 2012, Billett, 1999). Interestingly, when asked a direct question about their learning, the professionals tended to nominate structured learning activities such as face-to-face and online courses as their key learning experiences. In contrast, the critical incident style questions (Flanagan, 1954) resulted in the professionals describing their learning as being a process of trial and error, often assisted by others or online resources, through the course of doing their work. This highlights the disparity, previously noted by Boud and Middleton (2003), between how professionals experience learning through work yet name their learning as being structured. This study differs in investigating experiences of both work and learning simultaneously which provides context, and potential explanations, for why learning is experienced in this way.

The nature of work within complex adaptive organisations was the second category which emerged from the study. This category describes how work was experienced as fluid and influenced by varying degrees of emergence, agency, complex social networks, and adaptation. The complex adaptive organisations conceptual framework was used particularly in analysing the data relating to fluid work to investigate how work was experienced within 
the broader context of the organisational system where adaptation and emergence featured as important elements of the experience of work. The professionals described work that was constantly changing, requiring high levels of adaptation which required learning new skills and developing relationships. As a systems analyst from a member services organisation commented,

Everything changes now. You kind of just go bend with everything and go with the flow with whatever's happening.

Overall, the professionals interviewed identified their work as highly changeable, time pressured, and that it required constant adaptation to emerging requirements. A scientist/manager from a government scientific organisation described this well when he noted that he was always learning.

You apply for a promotion, you don't get it, you learn about it. Every new process that you have to implement that you learn about, every new initiative that comes along.....you're always learning.

Descriptions of learning as a continuous process of adaptation were common across the professionals. For example, an administrative assistant from a pharmaceutical company noted that,

...things change all the time, so you've got to be able to adapt quickly and to be able to share that knowledge and skills too.

This excerpt highlights the relationship which was found between change within the organisation (here described as emergence) and adaptation. Emergence and adaptation played key roles in the fluidity of work and subsequent experiences of learning.

Complex, inter-related social networks were also consistently identified by the professionals interviewed as a key method of both learning and getting one's job done. A radio producer 
for a public broadcaster, for example, highlighted the necessity of nurturing relationships amongst her network in order to influence others so that they would assist her.

... it's those relationships that you're developing with others and how you're able to influence others in regards to say, assisting you with the job or giving information from them so you can hone your job in that way.

The professionals consistently described seeking out colleagues within their team or professional networks to learn from, or to ask them for an introduction to someone with more specific expertise. This highlighted the professionals' experiences of work and learning as relational phenomena, supporting the broader 'turn' within the workplace learning literature towards describing learning as relational (Reich and Hager, 2014, Hopwood, 2016, Gherardi et al., 1998). A complex adaptive systems view of organisations highlights the importance of this relationality by foregrounding the role of complex social networks within organisations. The final key defining element of complex adaptive organisations is agency. All of the professionals interviewed exercised agency within their work, with flexibility to determine their daily activities within the boundaries of their area of responsibility. These boundaries influenced varying degrees of agency depending on the positioning of the job within the organisation. The degree of agency which the professionals were able to exercise contributed strongly to the fluidity they experienced in their work with multiple, and often competing, demands placed on them. As stated by an executive assistant to the general manager of a busy marketing department,

I'm almost like an all-in-one one shampoo, conditioner and body wash.....I report directly to the GM [General Manager]. I look after him. I also support the team. We have a team administrator as well.....I kind of help her along with stuff as well.....And then I sit on lots of projects and I also look after a program that we 
run.....and then I help support administration on a digital project that we have as well.

In using the metaphor of being "an all-in-one shampoo, conditioner and body wash" she encapsulates not only the fluidity of her work, but also her agency in negotiating how this work is carried out.

The third category of description described how professionals' experiences of learning are influenced by the degree of fluidity experienced in their work. The data analysis indicated that the greater the degree of work fluidity, the greater the impetus towards learning through work. Learning through work appeared to be the most efficient way for professionals to learn and adapt at the speed required in their complex work context. In order to learn and adapt to emergence within the organisation, the professionals needed to operate within and across networks, and exercise agency in doing so.

Overall, this connection between fluid work and learning was most vividly illustrated in the accounts of two categories of professionals: scientists $(n=2)$ and administration assistants $(n$ =2). These professionals, for different reasons, were unique in identifying their learning as being primarily through their day-to-day work when asked directly about their learning at work. In particular, they did not identify face-to-face courses as being "for them". Talking about this issue one scientist within a government scientific organisation noted,

...the biggest thing day to day is just trying to solve the problems in your research that you encounter.....and they're not really things that you can plan for in advance and put it in a development plan.

Both of these groups reported high levels of agency in their jobs, particularly the scientists. Moreover, both groups also reported high levels of adaptation required for their work. For the administration assistants this mainly took the form of their roles being highly reactive to 
the needs of others. For the scientists it was the result of there being few other ways to learn available to them such was the specificity of the knowledge and skills required for their work. These two groups also nominated their professional networks as being central to successfully working in their job and within their respective organisations.

The fourth category of description described how professionals experienced an organisational emphasis on structured learning despite also experiencing learning as primarily through work. This emphasis within organisations was wryly summarised by a manager from an airline as, ...if you're not in the classroom, you're not doing training.

While a number of professionals described organisational learning frameworks which attempted to promote learning through work, the way in which learning was measured through performance processes ultimately ensured that the professionals experienced learning in structured ways, such as face-to-face courses, which were more easily monitored by the organisation. As a human resources professional from a bank noted,

I would say where we have some of the less formal arrangements, I think we kind of put structures around them that really tip them back into being a formal arrangement. So for example we know that a lot of coaching takes place, but we also know that really only takes place when we are putting some structure around it.

This highlights a disconnection between how the professionals experience learning and the learning opportunities that are supported by the organisations studied. Professionals reported that the prevailing organisational policies and practices tended to privilege formality in learning over informality, either explicitly or implicitly. This reveals opportunities for organisations to improve learning practices in order to better facilitate, and recognise, learning and adapting through fluid work. 


\section{Discussion}

Collectively, the four categories of description discussed above indicate that learning was experienced by professionals in complex adaptive organisations as being primarily through work and that this is strongly influenced by the degree of work fluidity experienced. The degree of work fluidity was influenced by adaptation, emergence, complex social networks, and agency which, in turn, influenced more flexible learning responses from the professionals. Despite this, the professionals reported that organisations were still providing, and recognising, structured learning initiatives as the primary source of learning at work.

This study raises many interesting issues relevant to work and learning researchers. Within the space constraints of this article two key implications for work and learning researchers are discussed - the importance of emergence and fluid work in considering work and learning at an individual level within an organisational system.

\section{Emergence}

As previously discussed, emergence has been increasingly taken up in workplace learning research, particularly as a metaphor (Hopwood, 2016) juxtaposed with earlier metaphors of learning at work around acquisition and transfer, and participation (Hager, 2011, Boud and Hager, 2012). However, in this study, using emergence as a defining element of complex adaptive organisations offered significant explanatory power for experiences of work and learning. This was achieved by defining emergence as a characteristic of the organisation rather than solely a metaphor for learning. Through this analysis, the unpredictability of emergence became apparent. Emergence was experienced by the professionals as producing the conditions for fluid work, requiring high levels of agency and adaptation, negotiation of complex social networks, and flexible learning approaches. Previous research has suggested that there is a greater need to look at the increasingly dynamic and unpredictable contexts 
where learning at work occurs (Unwin et al., 2007, Fuller et al., 2007). Understanding emergence as a key characteristic of organisations, and an important influence on work and learning within them, provides a way to focus on these unpredictable contexts.

\section{Fluid work}

Strongly linked to emergence is the concept of fluid work. The second issue of interest to work and learning researchers is that, by framing the study using complex adaptive organisations, the concept of fluid work and the influence that this has on experiences of learning emerged. It suggests that a more effective way to discuss learning affordances in the workplace is to examine the nature of the work and the work context specific to each professional. For example, the excerpts from administrative assistants above are an illustration.

Harteis et al. (2015) found in their study of the impact of age, gender, and occupation on workplace learning that occupational level was the only one of these where significant differences in learning affordances were found. They found that those in lower status occupations had a lower level of perceived learning support at work whereas higher status occupations, such as managers and supervisors, were offered a "more learning-conducive workplace environment than lower-status occupations" (Harteis et al., 2015). Interestingly, at first glance, this finding seems replicated in this study in terms of the administrative assistants identifying that the learning opportunities provided by the organisation were not applicable to them and they strongly identified that they learned mainly, and continuously, through their work. Recent work by Cho and Kim (2016) also reported a high level of informal learning on the part of administrative assistants influenced by their perceived job efficacy. The study reported here, however, showed that the work context of administrative assistants was one in which they possessed a high degree of agency within an environment of rich, learning-conducive work and interactions. This sort of context is one that researchers 
such as Billett (2001) and Eraut (2004) have noted is important for affording learning at work. This is further supported by the scientists' data which indicated that they also worked within contexts which were rich in interactions and learning-conducive work coupled with a high degree of agency. Arguably, scientists are a higher status occupation than administrative assistants yet they described their experiences of work and learning in very similar terms.

Previous workplace learning research, such as Billett's (2001) work on workplace affordances and Fuller and Unwin's (2003) continuum of expansive and restrictive practices, has demonstrated the importance of participation as a key element of learning at work and how workplace practices support or restrict this learning. This study, through the lens of the complex adaptive organisations conceptual framework, takes up this work by offering a framework to investigate both workplace affordances for learning from an organisational perspective as well as investigating how individual experiences of the work itself influence learning. The emphasis on structured learning by the organisations in this study particularly highlights that organisations appear slow to adapt their workplace learning practices to the challenges of the emerging and fluid work experienced by their employees. This suggests that, just as more flexible learning approaches are needed from individuals, organisations also need more flexible and adaptable workplace learning practices.

\section{Conclusion}

This article reported an empirical study of the work and learning experiences of professionals that innovatively used complex adaptive systems theory and an adapted phenomenographic approach to conceptualise and analyse the interview data. The analysis showed that professionals in complex adaptive organisations experience learning as primarily through 
work, strongly influenced by the fluidity of work. This experience was in contrast to their experiences of learning afforded by the organisations which were predominantly structured.

Two key conceptual developments which emerged in the study discussed here was the usefulness of understanding emergence as a key element of the organisation, allowing for a more rigorous use of emergence in addition to metaphorical usages. Secondly, related to emergence, the conceptual framework highlights the importance of fluid work in experiences of learning and how work fluidity is driven to a large degree by emergence within the organisation.

The challenges faced by individuals and organisations in the current context of the constantly shifting, globalised economy require a disruption of former ways of conceptualising work and learning. The study discussed here shows an innovative way forward in considering the fluid nature of contemporary work and the ways in which this influences learning through the lens of complex adaptive systems. 


\section{References}

Ashworth, P. (1999). "Bracketing" in phenomenography: Renouncing assumptions in hearing about student cheating. International Journal of Qualitative Studies in Education, 12, 707-721.

Ashworth, P. \& Lucas, U. (2000). Achieving empathy and engagement: A practical approach to the design, conduct and reporting of phenomenographic research. Studies in Higher Education, 25, 295-308.

Bailey, M. (2015). Professional development of HR practitioners - a phenomenographic study. European Journal of Training and Development, 39, 220-238.

Billett, S. (1999). Guided learning in the workplace. In: Boud, D. \& Garrick, J. (eds.) Understanding Learning at Work. London: Routledge.

Billett, S. (2001). Learning through work: Workplace affordances and individual engagement. Journal of Workplace Learning, 13, 209-214.

Billett, S. (2004). Workplace participatory practices. Journal of Workplace Learning, 16, 312-324.

Billett, S. (2011). Subjectivity, self and personal agency in learning through and for work. In: Malloch, M., Cairns, L., Evans, K. \& O'connor, B. N. (eds.) The SAGE Handbook of Workplace Learning. London: SAGE Publications Ltd.

Billett, S. \& Smith, R. (2010). Personal agency and epistemology at work. In: Billett, S., Fenwick, T. \& Somerville, M. (eds.) Work, Subjectivity and Learning: Understanding Learning through Working Life. Dordrecht: Springer Netherlands.

Boud, D. \& Hager, P. (2012). Re-thinking continuing professional development through changing metaphors and location in professional practices. Studies in Continuing Education, 34, 17-30.

Boud, D. \& Middleton, H. (2003). Learning from others at work: Communities of practice and informal learning. Journal of Workplace Learning, 15, 194-202.

Chiva, R., Ghauri, P. \& Alegre, J. (2014). Organizational learning, innovation and internationalization: A complex system model. British Journal of Management, 25, 687-705.

Chiva, R., Grandío, A. \& Alegre, J. (2010). Adaptive and generative learning: Implications from complexity theories. International Journal of Management Reviews, 12, 114-129.

Cho, H. J. \& Kim, J.-M. (2016). Administrative assistants' informal learning and related factors. Journal of Workplace Learning, 28.

Davis, B. \& Sumara, D. (2006). Complexity and Education: Inquiries into Learning, Teaching and Research, Mahwah, NJ, Lawrence Erlbaum and Associates.

Davis, C. (2012). Practice as complexity: Encounters with management education in the public sector. In: Hager, P., Lee, A. \& Reich, A. (eds.) Practice, Learning and Change: Practice-Theory Perspectives on Professional Learning. Dortrecht: Springer.

Dietz, T. \& Burns, T. R. (1992). Human agency and the evolutionary dynamics of culture. Acta Sociologica, 35, 187-200.

Eraut, M. (2004). Informal learning in the workplace. Studies in Continuing Education, 26, 247-273.

Eraut, M. (2007). Learning from other people in the workplace. Oxford Review of Education, 33, 403422.

Fenwick, T. (2008). Workplace learning: Emerging trends and new perspectives. New Directions for Adult \& Continuing Education, 17-26.

Fenwick, T. (2012). Complexity science and professional learning for collaboration: A critical reconsideration of possibilities and limitations. Journal of Education and Work, 25, 141-162. 
Fenwick, T. \& Dahlgren, M. A. (2015). Towards socio-material approaches in simulation-based education: Lessons from complexity theory. Medical Education, 49, 359-367.

Fenwick, T., Edwards, R. \& Sawchuk, P. (2011). Emerging Approaches in Educational Research: Tracing the Socio-material, London, Routledge.

Flanagan, J. C. (1954). The critical incident technique. Psychological Bulletin, 51, 327-358.

Fuller, A. \& Unwin, L. (2003). Learning as apprentices in the contemporary UK workplace: Creating and managing expansive and restrictive participation. Journal of Education and Work, 16, 407-426.

Fuller, A., Unwin, L., Felstead, A., Jewson, N. \& Kakavelakis, K. (2007). Creating and using knowledge: An analysis of the differentiated nature of workplace learning environments. British Educational Research Journal, 33, 743-759.

Gherardi, S., Nicolini, D. \& Odella, F. (1998). Toward a social understanding of how people learn in organizations. Management Learning, 29, 273-297.

Giddens, A. (1984). The Constitution of Society, Cambridge, Polity Press.

Hager, P. (2011). Theories of workplace learning. In: Malloch, M., Cairns, L., Evans, K. \& O'connor, B. N. (eds.) The SAGE Handbook of Workplace Learning. London: SAGE Publications Ltd.

Harteis, C., Billett, S., Goller, M., Rausch, A. \& Seifried, J. (2015). Effects of age, gender and occupation on perceived workplace learning support. International Journal of Training Research, 13, 64-81.

Holland, J. H. (1995). Hidden Order: How Adapation Builds Complexity, Reading, MA, AddisonWesley.

Holland, J. H. (2006). Studying complex adaptive systems. Journal of Systems Science and Complexity, 19, 1-8.

Hopwood, N. (2014). Four essential dimensions of workplace learning. Journal of Workplace Learning, 26, 349-363.

Hopwood, N. (2016). Professional Practice and Learning: Times, Spaces, Bodies, Things, Dordrecht, Springer.

Jacobson, M. J., Kapur, M. \& Reimann, P. (2016). Conceptualizing debates in learning and educational research: Toward a complex systems conceptual framework of learning. Educational Psychologist, 51, 210-218.

Jacobson, M. J. \& Wilensky, U. (2006). Complex systems in education: Scientific and educational importance and implications for the learning sciences. Journal of the Learning Sciences, 15, 11-34.

Johnsson, M. C. \& Boud, D. (2010). Towards an emergent view of learning work. International Journal of Lifelong Education, 29, 359-372.

Kersh, N. (2015). Rethinking the learning space at work and beyond: The achievement of agency across the boundaries of work-related spaces and environments. International Review of Education, 61, 835-851.

Lancaster, J. (2012). The complex systems of practice. In: Hager, P., Lee, A. \& Reich, A. (eds.) Practice, Learning and Change: Practice-Theory Perspectives on Professional Learning. Dortrecht: Springer.

Manidis, M. \& Scheeres, H. (2013). Practising knowing: Emergence(y) teleologies. Educational Philosophy and Theory, 45, 1230-1251.

Marton, F. (1994). Phenomenography. In: Husen, T. \& Postlethwaite, T. N. (eds.) International Encyclopedia of Education. 2nd ed. London: Pergamon. 
Marton, F. \& Booth, S. (1997). Learning and Awareness, Mahwah, NJ, Lawrence Erlbaun Associates.

Mason, M. (2008). What is complexity theory and what are its implications for educational change? Educational Philosophy \& Theory, 40, 35-49.

Milgram, S. (1967). The small world problem. Psychology Today, 2, 60-67.

Mitchell, M. (2009). Complexity: A Guided Tour, New York, Oxford University Press.

Newman, M. E. J. (2010). Networks: An Introduction, Oxford, Oxford University Press.

Palys, T. 2008. Purposive sampling. In: Given, L. M. (ed.) The SAGE Encyclopedia of Qualitative Research Methods. Thousand Oaks, California: SAGE Publications, Inc.

Reich, A. \& Hager, P. (2014). Problematising practice, learning and change: Practice-theory perspectives on professional learning. Journal of Workplace Learning, 26, 418-431.

Richardson, K. A. (2008). Managing complex organisations: Complexity thinking and the science and art of management. Corporate Finance Review, 13, 23-29.

Sawyer, R. K. (2005). Social Emergence: Societies as Complex Systems, Cambridge, Cambridge University Press.

Trigwell, K. (2000). A phenomenographic interview on phenomenography. In: Bowden, J. A. \& Walsh, E. (eds.) Phenomenography. Melbourne: RMIT University Press.

Tsoukas, H. \& Hatch, M. J. (2001). Complex thinking, complex practice: The case for a narrative approach to organizational complexity. Human Relations, 54, 979-1013.

Unwin, L., Felstead, A., Fuller, A., Bishop, D., Lee, T., Jewson, N. \& Butler, P. (2007). Looking inside the Russian doll: The interconnections between context, learning and pedagogy in the workplace. Pedagogy, Culture \& Society, 15, 333-348.

Watts, D. \& Strogatz, S. (1998). Collective dynamics of 'small-world' networks. Nature, 393, 440442.

Yates, C., Partridge, H. \& Bruce, C. (2012). Exploring information experiences through phenomenography. Library and Information Research, 36, 96-119. 